\title{
The value of nursing: a literature review
}

Khim Horton, Verena Tschudin, Armorel Forget

Address for correspondence:

Khim Horton, Faculty of Health and Medical Sciences, University of Surrey, Guildford, GU2 7TE, UK. Telephone: +44 (0)1483 68 5555, Fax: +44 (0)1483 68 6711. e-mail:

k.horton@surrey.ac.uk 


\begin{abstract}
Key words:

Globalisation, professional identity, recruitment and retention, value of nursing

This article is part of a wider study of the Value of Nursing and contains the literature search from electronic databases. Key words for the search included 'values of nursing', 'values in nursing', 'organisational values' and 'professional identity'. Thirty-two primary reports written in English between 2000 and 2006 were identified. The findings highlight the importance of understanding values and their relevance in nursing and how values are constructed. The value of nursing is seen to be influenced by cultural change, globalisation, advancement in technology and medicine. These factors are crucial in providing a more structured and measured view of what nursing is, which will result in greater job satisfaction among nurses, greater nurse retention and enhanced patient care within a supportive and harmonious organisation. The findings of this review have implications for policy makers in recruitment and retention and determining the global value of nursing.
\end{abstract}




\section{Introduction}

Much attention by governments is concerned about health care. Within nursing, the focus on recruitment and retention has often been on economic and demographic factors, such as terms and conditions of employment. The inadequate attention to the cultural, organisational, and professional values that may underlie such factors demonstrates a lack of understanding of these values and their importance. To have a map of various perceptions, whether positive or negative, of the values and meanings of, and in, nursing as a social/work/professional role would assist policy making in healthcare and healthcare education. For example, it is well recognised that levels of salary are not always critical factors in choice of profession and subsequent job-satisfaction.

Demographic developments, especially population ageing and the decline in fertility, as well as a variety of medical and bio-technological developments, have served to accentuate the attention given to care, raising political, financial and ethical issues and dilemmas that seem to reach into every corner of modern life. ${ }^{1}$ In the United Kingdom, the values in the National Health Service (NHS) have received significant attention culminating in three major. reports. ${ }^{2-4}$ Hewison $^{5}$ argued that NHS values are 'not fixed and the predominance of particular values chance over time' (p. 253). Adapting to the dynamic nature of health care is a challenge for nursing, and represents a value that is often not recognised by lay people, health professionals and governments. Malone ${ }^{6}$ argues that effective patient care requires a valued and rewarded nursing workforce.

With the continuing change in status and role of the nurse, the profession needs to question the direction in which it is heading, ${ }^{7}$ decipher its core values and reach some agreement about the moral nursing values of practice. ${ }^{8}$ It is of profound interest to the profession as a whole to continue to examine the ways in which the values in nursing are portrayed, and whether these are subject to change globally. This article provides background understanding of the current knowledge of the value of nursing. An integrative review has been undertaken to assess the current research available.

As the broadest type of review method, an integrative review allows its findings simultaneously to include experimental and non-experimental research in order to understand a phenomenon of concern, and incorporates a wide range of purposes: to define concepts, review theories and evidence, and to analyse methodological issues of a particular topic. ${ }^{9,10}$

\section{Aims and objectives}

The literature review was part of the second stage of the study 'The Value of Nursing' that has been ongoing for some years at the International Centre for Nursing Ethics at the University of Surrey, UK. This is an international study considering aspects of the value of nursing as well as values in nursing.

The aim of the review was to establish whether the global value of nursing has changed in the past five years, and if so, in what way. Change(s) in the value of nursing can impact on social identity of nurses and nursing, as well as the perceived satisfaction in the occupation chosen, and factors that will affect recruitment and retention. 
Our review questions were:

- What is the value of nursing?

- How has this changed in the past five years?

- What factors influence the value of nursing?

- What are the implications for nursing in a global context?

\section{Conceptualising Value}

Because we wanted to know about the value of nursing, we need to know what the word 'value' means. Value indicates what is important, worthwhile and worth striving for. It has often been suggested that individual achievement and materialism are major values in western industrial society. Like norms, values vary from society to society. Haralambos and Holborn ${ }^{11}$ give examples of western society's values placed on human life as expressed in terms of the norms associated with hygiene (in the home), of settling arguments or disputes without violence, protecting life and limb and the many safety regulations in the workplace. Lawton ${ }^{12}$ suggests that values are also beliefs that are considered to be socially and personally desirable and therefore are recognised as being important in organisations. Hewison ${ }^{5}$ adds that societal, organisational and personal values all influence the way people operate in large institutions.

In an early meeting of the working group of the 'Value of Nursing' research team, group members brainstormed what is meant by 'value of nursing'. Examples given were the estimation of nursing as a social and/or work and/or professional role in the minds of various stakeholders such as nurses, healthcare managers, physicians and other professionals, citizens, policy makers, current patients and nurse educators. 'Value' could be explicated in terms of degrees between polar opposites such as, for example, socially useful/useless, prestigious/low-status, fulfilling/unfulfilling, skillful/unskilled, respected/disrespected, opportunities for advancement/dead-end, requiring certain (named) virtues/not requiring certain (named) virtues, supported/unsupported, autonomous/dependent and so forth.

The research study itself was therefore conducted by interview, in order to decrease the potential for the researchers' own preconceptions to influence the outcome. Findings of the research will be published in due course.

\section{Method}

In order to be included in the literature review, studies had to

- have a focus on the value(s) of nursing, value in nursing, organisational value or professional identity

- be published during 2000-2006,

- be in English.

- be randomised controlled trials, observational studies, evaluation studies, or inductive studies. 
- be written by nurse authors from all branches, trained or student nurses.

- be inclusive of any setting (hospital, primary care and care homes)

- measure or self-report on the value of nursing, organisational value, or professional identity.

\section{Identification of studies}

We searched for all potentially relevant literature, both published and unpublished and included relevant evidence regardless of the country of origin. The search was carried using the research databases Medline, Cumulative Index of Nursing and Allied Health Literature (CINAHL), PsycINFO, Psychological and Behavioural Sciences Collection, Internurse and British Nursing Index. The search was restricted to English language items or pragmatic purposes, and published during 2000 -2006 to ensure currency. Key words used to identify relevant sources were

- value of nursing

- values in nursing

- professional identity

- organisational values

In addition, reference lists of papers, reports and review articles were checked to see if they cited references not identified by the electronic searches, and we contacted researchers and experts in the area to identify unpublished or grey literature. Using these criteria, a total of 121 articles were retrieved. Although titles and abstracts seemed to indicate that the retrieved articles were relevant, after reading it was found that 89 were not pertinent to this study. Appendix 1 details the retrieved articles, their design and methodologies.

\section{Study selection and data extraction}

All citations identified were downloaded and printed. Two authors independently screened titles and abstracts of citations identified by the electronic search, applied the selection criteria to potentially relevant papers and extracted data from the full papers onto our data. Disagreements were resolved by discussion with the third author.

Further work was then undertaken to select items where the title or abstract (where available) indicated that it related to key terms. Having retrieved the original articles, we scanned the bibliographies for references that also related to the value(s) of nursing. The search was halted when 'saturation point' was reached, that is, no new references appeared in the bibliographies of the most recent items. In a number of areas, especially in commentaries, it was evident from the search of bibliographies that a limited numbers of articles existed on our key words. In these cases, a representative sample, judged by the first two authors, was obtained. This review does not therefore claim to be exhaustive. However, the authors felt confident, given the number of times selected articles were cited in bibliographies, that all the key articles in the field were included. Items selected for review included empirical research. No item was excluded for methodological limitations, although these are discussed below. In total, 121 articles were gathered for review. 


\section{Procedure}

Each article was briefly read by one of the authors for main points before assigning it with key words. As advocated by Cooper, ${ }^{13}$ we reviewed, categorised and critiqued each item. To facilitate this process a code sheet was constructed to record pertinent information about the study.

The snowball method was used to identify additional literature, through the reference lists of research accessed through the databases. Relevant articles were then identified using the same process of database searching, first by title, then abstract and conclusion, followed by full text. These methods follow the recommendations of Conn et al. ${ }^{14}$

Following this initial review, articles were read in more detail and assessed by all three authors. The reading was supplemented by discussions regarding the findings of the review and the emerging themes. The findings are presented below using key themes. The country of origin of the individual studies cited below is indicated where appropriate.

Information of study design and some comments are given in the Appendix, with a summary of the findings in Table 1 . (Table 1 about here)

\section{Summary of Research}

The articles retrieved highlighted moral values as an integral part of society and of nursing. The literature also showed that this is an area of interest to researchers worldwide.

The research conducted in the 32 reviewed articles was based on data from 14 countries. A range of methods have been identified, including ethnography, phenomenology, qualitative interview, cross-sectional surveys and secondary analysis. The methods chosen to investigate the questions were appropriate to the stated purpose of the research. The studies used a variety of sampling methods, all with a precise definition as to why they were chosen. Most had descriptions of limitations of the study. These were mainly sample and design issues, including small sample size, convenience sampling, limited collection in one setting and limitations of cross sectional design.

The main themes that emerged and that have been used here to present the literature are

- The role of the nurse

- The concept of 'care'

- The concept of value

- Values and culture

- Moral values and the impact on nursing

- Personal value

- Organisational values

- Factors influencing the value of nursing 


\section{The role of the nurse}

The role of the nurse has always had its roots in moral values, often being considered a vocation. ${ }^{5}$ However, in the modern world nursing is no longer a predominantly religious profession. ${ }^{15}$ The 'nurse' is now defined as 'a person, often a woman, who is trained to tend the sick and infirm, assist doctors etc' ${ }^{16}$ and as someone that 'assists patients under the direct supervision of medical officers'. ${ }^{17}$

Thupayagale and Dithole ${ }^{18}$ argued that for many years nursing struggled 'with an inner hunger; a deep need for professional congruency and effectiveness' (p. 142). They added that the perception of many people, except those aligned to nursing, is to see nursing as an inferior and inadequate undertaking to be regarded as a 'profession'; drawing attention to its stagnation or lack of adequate recognition as a profession. Compared to other health disciplines such as medicine, pharmacy, and psychology, nursing is viewed as having a lower status. ${ }^{18}$ This demonstrates how the role of nursing is aligned with a particular 'value' compared to the other health disciplines.

The role of the nurse also includes having to deal with and adapt to a variety of situations and events including caring for people with long term illnesses and conditions, people at the end of life, health promotion, as well as fundamental care such as bathing, comforting and assisting patients and their relatives. ${ }^{15}$ It is therefore not surprising to find that the term 'nursing' is synonymous with caring for the sick and 'lame' ${ }^{15,19}$ Henderson $^{20}$ defined the unique function of the nurse as 'to assist the individual, sick or well, in the performance of those activities contributing to the health or its recovery (or peaceful death) that he would perform unaided if he had the necessary strength, will or knowledge. And to do this in such a way as to help him gain independence as rapidly as possible' ( $\mathrm{p}$. 15).$^{20}$ This role seems to have changed little, as illustrated by the UK Royal College of Nursing (p. 5), ${ }^{21}$ which defines nursing as 'the use of clinical judgment in the provision of care to enable people to improve, maintain, or recover health, to cope with health problems, and to achieve the best possible quality of life, whatever their disease or disability, until death'. Recent empirical studies on emotions and the therapeutic use of self in nursing observe that although physical and emotional closeness and intimacy are values promoted by the 'new nursing', relationships of 'emotional intensity' with their patients have not always been facilitated by nurses (p. 393). ${ }^{22}$

\section{The concept of 'care'}

Developments in the patterns of the delivery of care have been accompanied by renewed interest in the meaning and importance of care. ${ }^{23}$ Not surprisingly, care is increasingly understood as a topic of such fundamental social importance that its neglect or marginalization within sociology and related disciplines can no longer be defended. The concept of care also provides a perspective that cuts across a number of topics and levels of analysis, linking micro interactions and macro structures, drawing together formal and informal aspects of social relations rather than treating them as distinct or specialized topics. ${ }^{24-26}$ Chamberlayne and King (p. 8$)^{24}$ elaborate: 'Caring offers a doorway to the study of informal systems of welfare, to the extending of comparative social policy to the level, and to the transcending of the welfare regime approach'. 
In nursing it can be argued that 'emotions in nurse-patient relationships are managed through a form of knowing the patient, which creates a feeling of closeness but at the same time maintains a distance' that both parties are comfortable with. ${ }^{22}$ The intricate nature of caring in nursing has often been debated given that nursing has historically emphasized the need for nurses to separate their professional life from private emotions.

\section{The concept of value}

The literature highlights the integral role that values play in people's lives. Values determine a person's beliefs and actions. They can also be seen as being a much more fundamental part of human existence, with values directing the priorities we live by and shape our being in the world. ${ }^{27}$ In our literature review we found many aspects and definitions of values but very few articles gave specific definitions of the value of nursing. Many authors defined nursing through story telling, such as the RCN's publication of The Values of Nursing. ${ }^{28}$ We also found that 'respect' ${ }^{9-31}$ and 'caring' were often discussed, stating that they are the 'essence of the profession'. ${ }^{30,32,33}$ Within caring there is a philosophy of 'moral commitment towards protecting human dignity and preserving humanity' (p. 31). ${ }^{34}$ In Table 2 we have listed all other terms related to values in nursing found in the literature used. (Table 2 about here)

Because there is an assumption that values are determinants of social behaviour, it is important to understand the connection between personal values and job descriptions, ${ }^{35}$ especially if good outcomes are to be achieved..$^{33,35-37}$ Values influence job satisfaction, motivation and commitment, ${ }^{38}$ and consciously or unconsciously, values affect the way people act in their personal and professional lives. ${ }^{39}$

\section{Values and culture}

The link between values and culture is not surprising since values are influenced by a range of factors. ${ }^{37,43}$ For example, a person's culture has a large bearing on what value system he or she may adhere to since it shapes the ideas, values and belief systems to which a person is committed. ${ }^{44}{ }^{45}$ By culture we mean those aspects comprising geographical, historical, societal, linguistic and ethnic dimensions ${ }^{46}$ American culture is linked to individualism and self reliance, whereby an individual's rights are more important than those of society. Other cultures practice collectivism, whereby the need of society outweighs that of the individual. ${ }^{43}$ Hence, a person's individual value system emerges from the culture he or she associates with.

While the image of the nurse has many common features across cultures, popular portrayals of the nurse tend to be culture specific. ${ }^{15,36,37,47}$ Wros \& Doutrich (p. 138) (6) $^{36}$ highlight that Japanese and American nurses hold common values that are 'woven into the fabric of their patient care; they also have many practices that are based on their cultural background and reveal the heterogeneity of nursing across the two cultures'. Williams ${ }^{48}$ argues that an organisation's members will hold values that tend to be, on average, synonymous with those of their nation's culture. However, it is important to highlight that due to an increasingly global and multicultural society, the cultural values of individuals already residing within a country may vary substantially. This may also be reflected within work groups and organisations. ${ }^{43}$ 
All nursing codes have values embodied in them. In the United Kingdom, registered nurses are required to follow the Code of Professional Conduct. ${ }^{49}$ Interestingly, Japan and the USA have similar membership codes for nurses and therefore should subscribe to the same values. However, Japanese nurses value continuity, homogeneity, harmony, selfsacrifice and hard work, whereas USA nurses focus on control, diversity, patient treatment choice and self-protection. ${ }^{36}$ In some respect it can be argued that the value of nursing is diverse in a global sense, with geographical, regional and cultural influences. However, it is important here to note that for many years, Japanese (and Chinese) nurses were nurtured on textbooks of American and British bioethics with little consideration of difference in culture. ${ }^{50,51}$

\section{Moral values and the impact on nursing}

The dictionary ${ }^{16}$ definition of moral values is that they are "concerned with or relating to human behaviour, especially the distinction between good and bad or right and wrong behaviour'. As such, moral values have a great influence on the way nurses think and act and therefore play a major role in patient care. ${ }^{31}$ Conflict can occur when values differ, resulting in stress, anxiety, burn-out and even resignation. ${ }^{52}$ This can impact on the organisation, resulting in poor patient care and the failure to meets standards and targets. ${ }^{43}$ The importance of understanding the moral values in nursing was highlighted in a study by Naden and Eriksson ${ }^{31}$ who used a phenomenological-hermeneutic approach, collecting notes taken by four nurses, observations of the four nurses for up to four months, and interviews conducted with these nurses, as well as 30 patients on a medical and a surgical ward. Specific values found were courage, responsibility, respect, obligation, and moral attitude. Moral attitude leads to values being realised and can result in patients feeling positive about the care they receive. Nortvedt ${ }^{53} \mathrm{emphasized} \mathrm{the}^{2}$ importance of empathy and altruistic feelings in nursing and saw them as moral judgments stating that, "Values can be actualized to a large extent through a moral attitude that is characterized by sensitive and careful communication.' (p. 91). The understanding and concordance of moral values are therefore vital for the whole healthcare environment.

Nurses' values are linked to factors contributing to job satisfaction or dissatisfaction. ${ }^{39,43}$, ${ }^{54}$ When nurses become dissatisfied with work, they often distance themselves from patients, from nursing tasks, ${ }^{55}$ and from their inner-selves. ${ }^{56}$ A Greek study ${ }^{33}$ states that if employees were able to live out their moral values, they would experience job satisfaction, therefore resignation intention would decline. Such information indicates that if managers focus more on helping employees to attain their moral values, retention figures could increase. Butterworth et al.'s research ${ }^{57}$ indicated that nurses' occupational stress levels are increasing in England. The shortage of nurses and high staff turnover are seen to be compromising nurses' ability to provide the competent and compassionate care that is at the core of their moral value system. ${ }^{30-33}$ This also reflects the responsibility that nurses have towards patients, and why moral values are embedded in codes of conduct. ${ }^{58}$

\section{Personal value}


Research regarding value systems has shown that personal value systems influence professional lifestyle. ${ }^{35,38,39}$ In essence, when applied to nursing, this indicates that nurses' personal value systems influence the actions they take. This has many implications. If nurses are influenced by a strong value system, they will tend not to conform to conditions with which they disagree. ${ }^{59}$ This may also change the way in which nurses practice. Wros \& Doutrich ${ }^{36}$ clearly state that professional values differ between American, Chinese and Japanese nurses. Differing practices are apparent with regard to advocacy. Advocacy is a strong American value that is always considered when caring for patients, however in Japan nurses only advocate for their patients if asked to do so by the patients themselves. ${ }^{36}$

Begat et al., ${ }^{30}$ Fealy, ${ }^{15}$ McNeese-Smith \& Crook $^{27}$ and Perry ${ }^{39}$ all concur with the statement by WHO (p. 222) ${ }^{60}$ that to be able to 'meet the challenges of their profession, nurses need to be clear about why they think and act as they do, and they need to perceive themselves as being empowered'. This provides more evidence of the importance for nurses to have a clear understanding of what their values are.

From a nursing management perspective, understanding the values that motivate individual staff is an important tool for managers. In a survey of 412 nurses, McNeeseSmith \& Crook $^{27}$ reported a number of benefits to understanding these values and state that such knowledge can improve worker production, assist in team performance and cohesion and aid in performance counselling. These are all traits of the nursing profession that require attention. According to Fealy the 'good nurse ideal was inseparable from the values that constructed nursing practice and defined its boundaries'. (p. 654) ${ }^{15}$ Good nursing is defined by its values. It is therefore important for nurses to understand the values with which they practice, so that good nursing practice can be achieved.

\section{Organisational value}

Another important aspect relating to the value of nursing stems from organisational value. In the workplace, it is argued that an individual's values are shaped by the positions and responsibilities held, ${ }^{61}$ the effect of taking responsibility, achieving results, developing a sense of worth, and recognising and using skills and abilities. Buffon et $\mathrm{al}^{61}$ also looked at the values of an organisation and how these are determined. The most influential factors are: 'Integrity; respect; customer focus; involvement; quality; creativity/innovation; accountability and fairness'. (p. 248) ${ }^{61}$ Indeed, healthcare organisations and systems are prime examples of differing cultures within a working environment, and as such tend to have a variety of individual value systems. ${ }^{62}$ This in turn may lead to a range of individual professional goals and targets. Such an environment may have links to the feelings of 'being lost' and 'undervalued' that many nursing staff have communicated. ${ }^{63}$

\section{Factors influencing the value of nursing}

When seeking to understand what the global value of nursing is, it becomes evident that the western hemisphere is becoming much more individualistic and materialistic. ${ }^{31}$ The development of technology, especially information technology, has contributed to a rapid 
change in values, such as increasing the turnover rate of patients in hospital, patient focused care and nurses' job satisfaction. ${ }^{35}$

The study undertaken by Rognstad et $a l^{35}$ examines how Norwegian society has changed by comparing the values that modern nursing students hold to values evident 100 years ago. Using a quantitative longitudinal survey design Rognstad et al surveyed 301 nursing students and discovered that the once highly valued ideals of nursing, humility, solidarity and unity, have now been replaced by diverse ideas regarding life and expectations. They found that the key nursing values in today's culture were: freedom, individualism, positive acknowledgement and personal achievement. Rognstad et $a l^{35}$ attributed these differences in values to the changing culture and society, with particular reference to the developments of information technology.

Worldwide migration, changes in demographic patterns, varying fertility rates, increased numbers of multiracial and multiethnic individuals, and advanced technology all contribute to changes in cultural diversity and therefore influence values. However, it is important to recognize that cultures that influence values are not unique to geographic areas but are also part of institutions, e.g. the culture of a hospital. ${ }^{40-42}$

Globalisation results in people travelling further distances for business and pleasure and leads to an increase of a global market and an internationalisation of organisations. This in turn leads to increased migration figures across the world. ${ }^{64}$ This has a considerable impact on the daily work of nurses, with such realities as avian flu and international terror now a worldwide issue; such circumstances affect our health, our need for health services and the way in which nurses work. ${ }^{64}$ At the same time, the healthcare system is continuing to evolve. Medical advancement is based on developments in pharmacological and physiological research and the implementation of technology within all healthcare. ${ }^{62}$ In addition, the extension of nurses' role, including undertaking surgical procedures, performing venepucture as well as nurse prescribing, must have an impact on the value of nursing. ${ }^{65}$

\section{Discussion and implications for nursing}

Nurses have experienced great change in the last few decades in organisational and individual culture, which Begat et al. ${ }^{30}$ believe is 'putting extra pressure on nurses.' (p. 222). Such situations encourage nurses to have diverse life views and expectations, which can be truly ambivalent and heterogeneous, and influence their value system greatly. In multi-cultural societies, the cultural values of individuals already residing within a country can be substantial, and are reflected within certain work groups. ${ }^{35}$ These values also impact on the personal lives of nurses, influencing their decision making in family, religious and community environments.

There is currently very little research available on the value of modern nursing in a global context. Much of the literature in this review has highlighted the implicit need for understanding values, due to the fact that they underpin society, and more importantly to this study, to the way nurses interact with each other and with patients, and how satisfied they are with their job. Our study did not aim to consider values and patient outcomes or 
satisfaction, although this would be a logical sequence when investigating the value of nursing further.

Understanding the moral values in today's nursing will help nurses to work together with a common understanding of their aims. This should also allow greater appreciation of the practice of nursing, highlighting the equal importance of both fundamental basic nursing care, and the advanced clinical roles. However, when these values have been identified, a number of barriers hinder their inclusion into nursing practice. Fundamental or basic nursing care holds less of a position of importance within society compared to more technically advanced clinical nursing roles.

To overcome these many barriers, a shift in thinking will need to take place. Nursing unions are among the few organisations that hold the power and authority to influence such change. If nurses hold shared values, they themselves form a powerful group who can start to influence the thinking about and attitudes towards the nursing profession by policy makers and governments. We can only hypothesise how this may happen. The lack of research in these areas gives no information on how changes may be tackled and what the results may be. The only deduction we can make is that a cohesive nursing workforce will lead to greater job satisfaction and ultimately better patient care. This can only happen when nurses have a greater understanding of the role of nursing, and what it means. The appeal of nursing as a realistic and successful profession needs to be built, tackling the recruitment problem, and establishing a strong career-focused workforce.

The literature review has highlighted past values in nursing, and has also touched on the impact of developing technology in medicine, as well as the impact of culture and globalization. We echo Malone's ${ }^{6}$ appeal that 'promoting the value of nursing has to come from nurses themselves' in that we need to utilise the body of evidence to demonstrate the positive impact we have on patient care.

\section{Conclusions and implications for research}

\section{Limitations of the review}

We recognise that the difficulty with using an integrative review strategy is that the methods of analysis, synthesis and conclusion-drawing are poorly planned. This is because it endeavours to review many differing types of research, and incorporates many purposes. ${ }^{7,8}$ Despite the number of studies in the literature relating to the value of nursing, there are many gaps in the scientific base of knowledge related to male nurses, for instance. Male nurses have not been studied adequately because of the limitations in sampling design, with too few men in many samples. Many of the samples have been convenience samples, limiting the generalizability of the findings. Because of the small sample sizes and the predominantly female presence in nursing, analyses by gender has been limited.

There is also a need for longitudinal studies that analyze the changes that occur in the lives, perceptions, and needs of nurses over the duration of their career. Reports of such studies across countries would help to inform global policies in nursing recruitment and retention. Longitudinal studies into the value of nursing could answer questions that are 
not possible to be resolved by cross-sectional designs. Studies that explore differences in the value of nursing by gender and over time are needed.

Our colleagues have made us aware of studies that would have been as relevant as those included in the literature review, but because of the search carried out by specific keywords, they were not included. This is an indicator of the limitations of keywords and indeed of search databases. We add details of some of these studies to complement the above text. ${ }^{66-70}$

\section{Implications for policy makers}

The findings of this review have implications for policy makers at many levels. It is becoming increasingly important for governments to see health issues as being related to economic and foreign policies. ${ }^{71}$ Issues of national and international security and protection, and abuses of humanitarian law and human rights result in widespread illness and disease. Recruitment and retention and personnel - what type, how many, for which needs - have long been seen as vital aspects of policies in health care, with ripple effects on basic and secondary education, taxation and the general wellbeing of a society. For nurses to be involved in policy making at high levels, it is important that they understand clearly what the value of nursing is in terms of the wider global economy and planning for good governance.

Acknowledgements

We would like to thank the researchers of the Value of Nursing project. We thank in particular Dr Douglas Olsen, Professor Samantha Pang and Professor Janet Storch for their valuable comments on earlier drafts of this text.

Khim Horton, Post-Doctoral Research Fellow, Centre for Research in Nursing and Midwifery Education, European Institute of Health and Medical Sciences, University of Surrey, Guildford, UK

Verena Tschudin, Reader in Nursing Ethics, International Centre for Nursing Ethics, European Institute of Health and Medical Sciences, University of Surrey, Guildford, UK Armorel Forget, Staff nurse, Royal Surrey County Hospital, Guildford, UK 


\section{References}

1 Fine M. Individualization, risk and the body. Sociology and Care, Journal of Sociology 2005; 41: 247-266.

2 Hutton W. New Life for Health; the Commission on the NHS. London: Longman, 2000.

3 New B. A Good Enough Service: Values, Trade-Offs and the NHS. London: Institute for Public Policy Research/King's Fund, 1999.

4 Steele J Wasted Values: Harnessing the Commitment of Public Managers. London: Public Management Foundation, 1999.

5 Hewison A. Values in the National Health Service: implications for nurse managers. Journal of Nursing Management 2001; 9: 253-258.

6 Malone B. Promoting the value of nursing in the context of global shortage. International Nursing Review 2003; 50: 129-130.

7 Castledine G. Ten years on and I fear for the future of nursing. British Journal of Nursing 2002; 11: 518.

8 Castledine G. The benefits of putting spirit into an organization. British Journal of Nursing 2003; 12: 335.

9 Whittemore R, Knafl K. The integrative review: updated methodology. Journal of Advanced Nursing 2005; 2: 546-553.

10 Torraco RJ. Writing integrative literature reviews: guidelines and examples Human Resource Development Review 2005; 4: 356-367.

11 Haralambos M, Holborn M. Sociology: Themes and Perspectives. $4^{\text {th }}$ ed. Harper Collins, London, 1995.

12 Lawton A. Ethical Management for the Public Services. Buckingham: Open University Press, 1998.

13 Cooper H. Synthesizing research: a guide for literature reviews. $3^{\text {rd }}$ ed. Thousand Oaks, CA: SAGE, 1998.

14 Conn VS, Isaramalai S, Rath S, Jantarakupt P, Wadhawan R, Dash Y. Beyond MEDLINE for literature searches. Journal of Nursing Scholarship 2003; 35: 177-182

15 Fealy GM. 'The good nurse': visions and values in images of the nurse. Journal of Advanced Nursing 2004; 46: 649-656.

16 Collins English Dictionary and Thesaurus. Glasgow: HarperCollins Publishers, 2003. 
17 Questia Online Library http://www.questia.com/index.jsp?

CRID=nursingOFFID=selKEY=nursing, 2005 accessed 13/03/06

18 Thupayagale G, Dithole K. 2005 What is in a name: the case of nursing. Nursing Forum 2005; 40: 141-144.

19 Seymer LR. A general history of nursing. London: Faber \& Faber, 1957.

20 Henderson V. The nature of nursing: a definition and its implications for practice, research, and education. New York: Macmillan, 1966.

21 RCN Defining nursing. London: RCN, 2003. Available online from: http://www.rcn.org.uk/downloads/definingnursing/definingnursing-a5.pdf Accessed $13 / 5 / 06$.

22 Allan $\mathrm{H}$, Barber D. Emotional boundary work in advanced fertility nursing roles. Nursing Ethics 2005; 12: 391-400.

23 Feder EK, Kittay EF. Introduction IN Kittay EF and EK Feder (eds) The subject of care: feminist perspective on dependency. Lanham, MD: Rowman and Littlefield, 2002.pp1-13.

24 Chamberlayne P, King A. Cultures of care: biographies of carers in Britain and the two Germanies. Bristol: Policy Press,2000.

25 James N. Care organisation and physical labour and emotional labour. Sociology of Health and Illness 1992; 14: 488-509.

26 Hochschild AR. The Managed Heart: Commercialisation of Human Feeling. Berkeley, CA: University of California Press, 1983.

27 McNeese-Smith DK, Crook M. Nursing values and changing nurse workforce.Values, age, and job stages. Journal of Nursing Administration 2003; 33: 260-270.

28 Royal College of Nursing. The Values of Nursing. London:RCN, 1992.

29 MacIntosh J. Reworking Professional Nursing Identity. Western Journal of Nursing Research 2003; 25: 725-741.

30 Begat I, Ellefsen B, Severinsson E. Nurses' satisfaction with their work environment and the outcomes of clinical nursing supervision on nurses' experiences of well-being - a Norwegian study. Journal of Nursing Management 2005; 13: 221-230.

31 Naden D, Eriksson K. Understanding the importance of values and moral attitudes in nursing care in preserving human dignity. Nursing Science Quarterly 2004; 17: 86-91.

32 Gregg MF, Magilvy JK. Professional identity of Japanese nurses: bonding into nursing. Journal of Nursing and Health Sciences 2001; 3: 47-55. 
33 Lemonidou C, Papathanassoglou E, Giannakopoulou M, Patiraki E, Papadatou D. Moral professional oersonhood: ethical reflections during initial clinical encounters in nursing education. Nursing Ethics 2004; 11: 122-137.

34 Watson J Assessing and Measuring Caring in Nursing and Health Sciences. New York: Springer, 2002

35 Rognstad MK, Nortvedt P, Rognstad O. Helping motives in late modern society: values and attitudes among nursing students. Nursing Ethics 2004; 11: 227-239.

36 Wros PL, Doutrich D, Izumi S. Ethical concerns: comparison of values from two cultures. Journal of Nursing and Health Sciences 2004; 6: 131-140.

37 Yamaguchi S. Nursing culture of an operating theatre in Italy. Journal of Nursing and Health Sciences 2004; 6: 261-269.

38 Tzeng H M Satisfying nurses on job factors they care about. Journal of Nursing Administration 2002; 32: 306-09.

39 Perry B. Core nursing values brought to life through stories. Nursing Standard 2005; 20: $41-48$.

40 Wikipedia P.Culture 2006a. [online] Available from http://en.wikipedia.org/wiki/Culture Accessed 19/5/06

41 Nichols B. Globalisation. Imprint. February/March, 2006; 17.

42 Jeffreys M. Cultural Competence in Clinical Practice. Imprint. February/March 2006: 37.

43 Parkes LP, Bochner S. Person-organisation fit across cultures: an empirical investigation of individualism \& collectivism. Applied Psychology 2001; 50: 81-108.

44 Goopy SE. Taking account of local culture: limits to the development of a professional ethos. Nursing Inquiry 2005; 12: 144-154.

45 Chen YC. Chinese values, health and nursing. Journal of Advanced Nursing 2001; 6: $270-273$

46 Watson R, Hoogbruin AL, Rumeu C, Beunza M, Barbarin B, MacDonald J, McCready T. Differences and similarities in the perception of caring between Spanish and UK nurses. Journal of Clinical Nursing 2003; 12: 85-92.

47 Dombeck MT. Working narratives: gender and race in professional personhood. Research in Nursing \& Health 2003; 26: 351-365.

48 Williams LL. Impact of Nurses' Job satisfaction on Organisational Trust Health Care Management 2005; July-September, 203-211. 
49 Nursing and Midwifery Council. The NMC code of professional conduct: standards for conduct, performance and ethics. London: NMC, 2004.

50 Davies, AJ. Global influence of American nursing: some ethical issues. Nurs Ethics 1999, 6:118-126.

51 Izumi S Bridging western ethics and Japanese local ethics by listening to nurses' concerns. Nurs Ethics 2006, 13: 275-283.)

52 Varcoe C, Doane G, Pauly B, Rodney P, Storch JL, Mahoney K, McPherson G, Brown H, Starzomski R. Ethical practice in nursing: working the in-betweens. Journal of Advanced Nursing 2004; 45: 316-325.

53 Nortvedt P. Sensitive judgment: Nursing, moral philosophy and an ethics of care. Oslo: Tano Aschehoug, 1996.

54 Verplanken B. Value congruence and job satisfaction among nurses: a human relations perspective. International Journal of Nursing Studies 2004; 41: 599-605.

55 Demeronti E, Bakker AB, Nachreiner F, Schaufeli WB, A model of burnout and life satisfaction amongst nurses. Journal of Advanced Nursing 2000; 32: 454-64.

56 Cameron M. Ethical distress in nursing. Journal of Professional Nursing 1997; 5: 280.

57 Butterworth T, Carson J, Jeacock, J, White E. Stress, coping, burnout and job satisfaction in British nurses: findings from the clinical supervision evaluation project. Stress Medicine 1999; 15: 27-33.

58 Itzhaky H, Gerber P, Dekel R. Empowerment, skills, and values: a comparative study of nurses and social workers. International Journal of Nursing Studies 2004; 41: 447455.

59 Watson J.Nursing: seeking its source and survival. ICUs and Nursing Web Journal 2002; 9: 1-7.

60 World Health Organization. International nurse mobility; trends and policy implications. Geneva: WHO, 2003

61 Buffton B, Sullivan W, Sullivan R. Aligning individual and organizational values to support change. Journal of Change Management 2002; 2: 247-254.

62 Fagerberg I. Registered nurses' work experiences: personal accounts integrated with professional identity. Journal of Advanced Nursing 2003; 45: 284-291.

63 Waters A. Nursing is the most emotionally rewarding career. Nursing Standard 2005; 19: $22-28$.

64 Hirschfeld M. An international perspective In: Davis AJ, Tschudin V \& de Raeve L eds. Essentials of teaching and learning in nursing ethics; perspectives and methods. Edinburgh: Churchill Livingstone, 2006; 325-337. 
65 Casey J. Advanced nursing practice in renal medicine. Nursing Times, 2002. [online] Available from: http://www.nursingtimes.net/nav?

page $=$ nt.article $\&$ fixture article $=214132 \&$ type $=$ STORY $\&$ fixture $=$ ARTICLE $\&$ fixture $=$ FE ATURE\&resource $=214132 \&$ product $=$ NT $\&$ exactPhrase $=$ nursing + the + future $\&$ section $=\mathrm{A}$ RCHIVE Accessed 2/6/06

66 Pang MCS. Nursing Ethics in Modern China: Conflicting Values and Competing Role Requirements. Value Inquiry Book Series 140. Amsterdam-New York: Rodopi 2003.

67 Pang MCS, Wong TKS, Wang CS, Zhang ZJ, Chan YLH, Lam WYC. Towards a Chinese definition of nursing Journal of Advanced Nursing, 2004, 46(6), 657-670

68 Pang MCS, Sawada A, Konishi E, Olsen D, Yu PLH, Chan MF, Mayumi N, A Comparative Study of Chinese, American and Japanese Nurses; Perception of Ethical Role Responsibilities, Nursing Ethics, May, 2003, 10(3), 295-311

69 Pang MCS, Arthur D, Wong TKS. Drawing a qualitative distinction of caring practices in a professional context: The case of Chinese nursing. Holistic Nursing Practice 2000; 15: 22-31.

70 Wong, TKS, Pang, MCS. Holism and caring: Nursing in the Chinese health care context. Holistic Nursing Practice 2000; 15: 12-21.

71 Anonymous. Health is a foreign policy. (Interview with JH Støre) WHO news. Bulletin of the World Health Organization 2007; 85: 167-168.

72 Barron D, West E. Leaving nursing: an event-history analysis of nurses' careers. Journal of Health Service Research Policy 2005; 10: 150-57.

73 Cowin L. Measuring Nurses' self-concept. Western Journal of Nursing Research 2001; 23: 313-25.

74 Fagerberg I, Kihlgren M. Experiencing a nurse identity: the meaning of identity to Swedish registered nurses two years after graduation. Journal of Advanced Nursing 2000; 34: $137-145$.

75 Faithfull S, Hunt G. Exploring nursing values in the development of a nurse-led service. Nursing Ethics 2005; 12: 440-52.

76 Heung YYJ, Kwong WYE, To SST, Wong HCD, Wong KYF. Severe acute respiratory syndrome outbreak promotes a strong sense of professional identity among nursing students. Nursing Education Today 2005; 25: 112-118.

77 Martin P, Yarbrough S, Danita A. Professional values held by baccalaureate and associate degree nursing students. Journal of Nursing Scholarship 2003; Third Quarter: 291-296.

78 Nelson S, Gordon S. The rhetoric of rupture: nursing as a practice with a history? Nursing Outlook 2004; 52: 255-61. 
79 Pask EJ. Moral agency in nursing: seeing value in the work and believing that I make a difference. Nursing Ethics 2003; 10: 165-174.

80 Pellatt G. Perceptions of the nursing role in spinal cord injury rehabilitation. British Journal of Nursing 2003; 12: 292-99.

81 Shih F-J, Liao Y-C, Chan S-M. The impact of the 9-21 earthquake experiences of Taiwanese nurses as rescuers. Social Science and Medicine 2002; 55: 659-672.

82 Wimpenny P, Gault B, MacLennan V, Boast-Bowen L, Shepherd P. Teaching and learning about culture: A European journey. Nurse Education Today 2005; 25: 398-404.

83 Wong FKY, Lee WM. A phenomenological study of early nursing experiences in Hong Kong. Journal of Advanced Nursing 2000; 31: 1509-517. 